Review

\title{
Super-Resolution of Nano-Materials and Quantum Effects Obtained by Microspheres
}

\author{
Y. Ben-Aryeh *
}

Physic Department, Technion-Israel Institute of Technology, Haifa, 32000, Israel; E-Mail: phr65yb@physics.technion.ac.il

* Correspondence:Y. Ben-Aryeh; E-Mail: phr65yb@physics.technion.ac.il

Academic Editor: Sotirios Baskoutas

Special Issue: Quantum Confinement Effects in Nano Material

Recent Progress in Materials

2019 , volume 1 , issue 3

doi:10.21926/rpm.1903003
Received: July 29, 2019

Accepted: August 26, 2019

Published: September 04, 2019

\begin{abstract}
In this article, microsphere super-resolution, which are beyond the Abbe classical limit, are described. The conversion of evanescent waves into propagating waves is analyzed by using the geometry of the microsphere. In microsphere experiments, a nanojet is produced near the focal plane, where its width is smaller than the Abbe limit and remains unchanged in the axial direction for certain wavelengths. The interference between the evanescent waves being converted into propagating waves and the nanojet leads to an increase in light intensity and confinement effects in the focal plane. However, the nanojet is not the main source of the super-resolution as the fine structures are available mainly in the evanescent waves. Quantum effects for super-resolution are obtained from special properties of the evanescent waves leading to an uncertainty relation. Several methods to increase the phase contrast in microsphere experiments have been described, which can be used for phase object measurements. Plasmon interaction can be used for measuring fine structures of special systems and for converting evanescent waves into propagating waves but they might also change the optical image in a way which is difficult to analyze. Therefore, most microsphere high-resolution experiments were conducted without plasmon interactions.
\end{abstract}

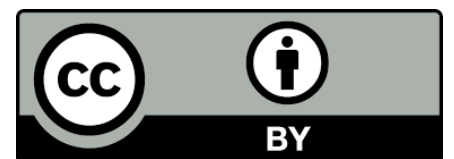

(C) 2019 by the author. This is an open access article distributed under the conditions of the Creative Commons by Attribution License, which permits unrestricted use, distribution, and reproduction in any medium or format, provided the original work is correctly cited. 


\section{Keywords}

Nano-materials; super-resolution; microsphere; confinement and quantum effects

\section{Introduction}

Ernest Karl Abbe approximated the diffraction limit of a microscope as

$$
d=\frac{\lambda}{2 n \sin \varphi}
$$

where $d$ is the resolvable feature size, $\lambda$ is the wavelength of light, $n$ is the index of refraction of the medium being imaged in, and $\varphi$ is the half-angle subtended by the optical objective lens (representing the numerical aperture) [1]. This results in the best outcome that can be achieved by conventional microscopy. As can be deduced from Abbe resolution limit, the resolution is increased by using "immersed lens," i.e., in which the objective is immersed in liquid with the index of refraction $n>1$ (quite often oil is used). The optical resolution of conventional lenses is limited to approximately $200 \mathrm{~nm}$ and thus they cannot be used to resolve nanostructures. Therefore, recently a 50-nm resolution nanoscope has been developed [2], that uses optical transparent microsphere (for example, microspheres of $\mathrm{SiO}_{2}$ with a diameter of 2-9 $\mu \mathrm{m}$ ) to overcome the white light diffraction limit. The microsphere nanoscope can be operated in both transmission and reflection modes and generate magnified virtual images with a magnification up to $8 \mathrm{X}$. This offered opportunities to image viruses and biomolecules in real-time. By using a microsphere confocal nanoscope, sub-diffraction imaging was carried out at $25 \mathrm{~nm}$ lateral resolution in the visible spectrum [3]. Several properties of microspheres, which are conducive for obtaining super-resolution, have been described in the literature. Usually by the use of microspheres, virtual images are obtained but they elicit real super-resolution using high refractive index [4]. In subsequent studies, the effects of the microsphere radius [5], near-field, and nanojet were analyzed [6]. A simple configuration, not involving resonances, was described [7] to reach an effective volume of $0.6(\lambda / n)^{3}$. Phase contrast in microsphere imaging can be increased by darkfield microscopy [8]. It was claimed that Mie resonances can lead to high microsphere resolution $[9,10]$. Dielectric sphere terahertz (THz) super-resolution imaging was described [11]. Application of the microsphere to focus laser light on samples which led to nano patterning was suggested [12, 13]. Quantization of evanescent waves was analyzed by which the propagation wavelength is reduced as a cooperative effect of several photons, increasing the resolution far beyond the Rayleigh criterion [14].

Later, many other experiments were carried out to delineate the properties of super resolution [15-28]. It was proposed that there must be some new mechanisms that could explain the unusual imaging properties [29]. It was also asserted that "previous studies adopted a phenomenological explanation mainly based on the super-focusing effect of a photonic nanojet" "but there must be some other physical mechanism" [30]. There have been debates and disagreements about the interpretation of the microsphere super-resolution. In the present review I would like to address the following four fundamental questions about microsphere super-resolution and explore the 
possible answers to these questions by developing theoretical analyses, which have been found in agreement with experiments:

a) What is the main source for microsphere super-resolution?

As analyzed in Section 2, the super-resolution is related to evanescent waves, in which the wave vector component in the longitudinal direction is imaginary and the spatial vector components in the transversal directions become large so that they can detect the fine structures of an object. Such effects are well known in the near-field scanning optical microscopy (NSOM) in which nanostructures are detected and they overcome the far-field resolution limit by exploiting the properties of evanescent waves. By using complex Snell's law and the special geometry of the microsphere described in Figure 1, the conversion of evanescent waves to propagating waves is analyzed in Section 2 and it is proved that this analysis concords well with the experimental results.

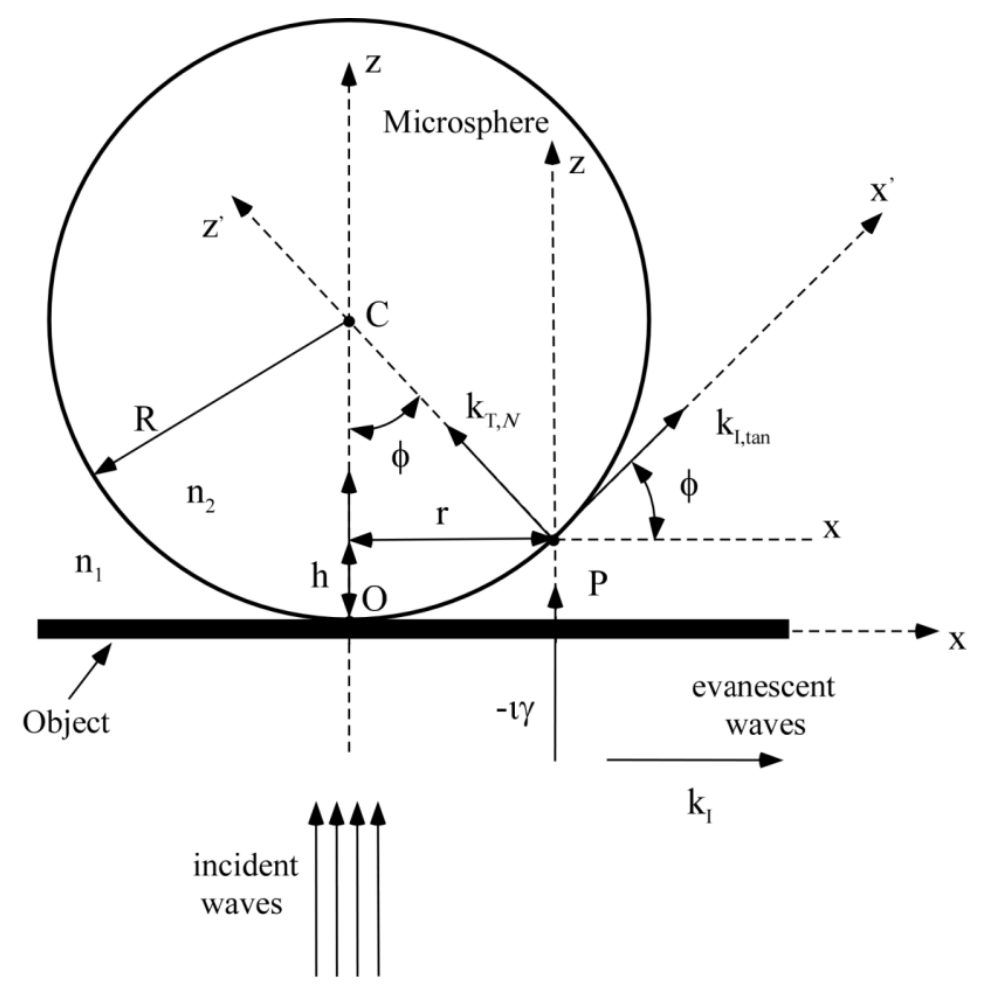

Figure 1 Conversion of evanescent waves to propagating waves. A dielectric microsphere with refractive index $n_{2}$ and radius $R$ is located above a thin film of the object at a contact point $O$ where the medium between the object and the microsphere has refraction index $n_{1}$. A plane EM wave is transmitted through the thin film of the object in the perpendicular direction and incident on the microsphere at point $P$. Evanescent waves with spatial vector $k_{I} \hat{x}-i \gamma \hat{z}$ propagate near the contact point $O$ (the subscript $I$ represents incident waves). The evanescent wave spatial vector is rotated by angle $\phi$ in the counterclockwise direction with a component $k_{I, \text { tan }}$ tangential to the microsphere surface at point $P . k_{T, N}$ represents the component of the spatial vector inside the microsphere, which is perpendicular to the microsphere surface. The decay of the evanescent waves at point $P$ is proportional to $\exp (-\gamma h)$ and this decay is small under the condition $r=R \sin \phi \ll R$. 
b) What is the role of quantum coherence in microsphere super-resolution?

As analyzed in Section 3, the evanescent waves introduce a quantum mechanical cooperation between $n$ photons reducing the wavelength to $\lambda / n$ and there is a distribution over different $n$ numbers. Following the Abbe resolution limit, we find that by reducing the effective wavelength, the resolution is improved due to quantum coherence. Further, fluorescence spectroscopy can lead to super-resolution, which is far better than those obtained in microsphere experiments [31]. However, the microsphere measurements include the phases of the object (proportional to the optical distance, i.e., to the geometric path multiplied by the index of refraction) in a way which is much better than fluorescence spectroscopy. The phase-contrast measurements [32], described in Section 5, have important applications especially in measuring semi-transparent biological systems.

c) What is the effect of plasmon interaction on microsphere super-resolution?

When there are two different groups of EM modes, Fourier series expansion can be used for each group of modes and the total EM field (in certain $x, y, z$ coordinate system) can be described by the products of the fields in the two separated parts of the system. In order to describe the boundary conditions with spatial modes, we need, however, to transform this product by inverse Fourier transform to convolution of the spatial modes of the separated parts of the system. Ebbesen and his colleagues showed [33-35] that a thin metallic grating with arrays of subwavelength holes can transmit light intensity at certain frequencies. This intensity is many orders stronger than the light intensity incident on the area of these holes. This effect was explored in our previous work [36] on the convolution between evanescent waves' momentum transfer function and momenta of surface-plasmons. It has been shown that the transmission by microspheres can be enhanced by inserting metallic films in the microsphere system [37] or by employing metal-coated microspheres [38]. As shown in Section 3, the plasmon interaction is quite complicated and might change the optical image in a way which is difficult to analyze. Therefore, it can be said that plasmon interaction including quantum effects [39] is a different field from the present one.

d) What is the role of the nanojets in microsphere super resolution?

The evanescent waves that are converted to propagating waves are transmitted near the symmetry axis of the microsphere. These waves interfere with propagating waves that enter the microsphere at distances which are far from the contact point $O$ and they do not include the evanescent waves (converted to propagating waves). They produce the nanojet effect as described in Figure 2.

The scattering of light from small spherical particles with a dimension larger than wavelength has been described by Mie theory. Photonic nanojets emerge as narrow and elongated spots with high field enhancement. The bandwidth of a nanojet can be smaller than the classical diffraction limit given by Eq. (1), in fact as: $\sim \lambda / 4$ for microspheres. Its width remains unchanged in the axial direction for some wavelengths. Mie theory solves Maxwell's equations for the particular geometry and numerous numerical and experimental studies have been reported on nanojets [4048]. Some studies have especially been focused on the influence of the photonic nanojet of microsphere on microsphere imaging [49-56]. 


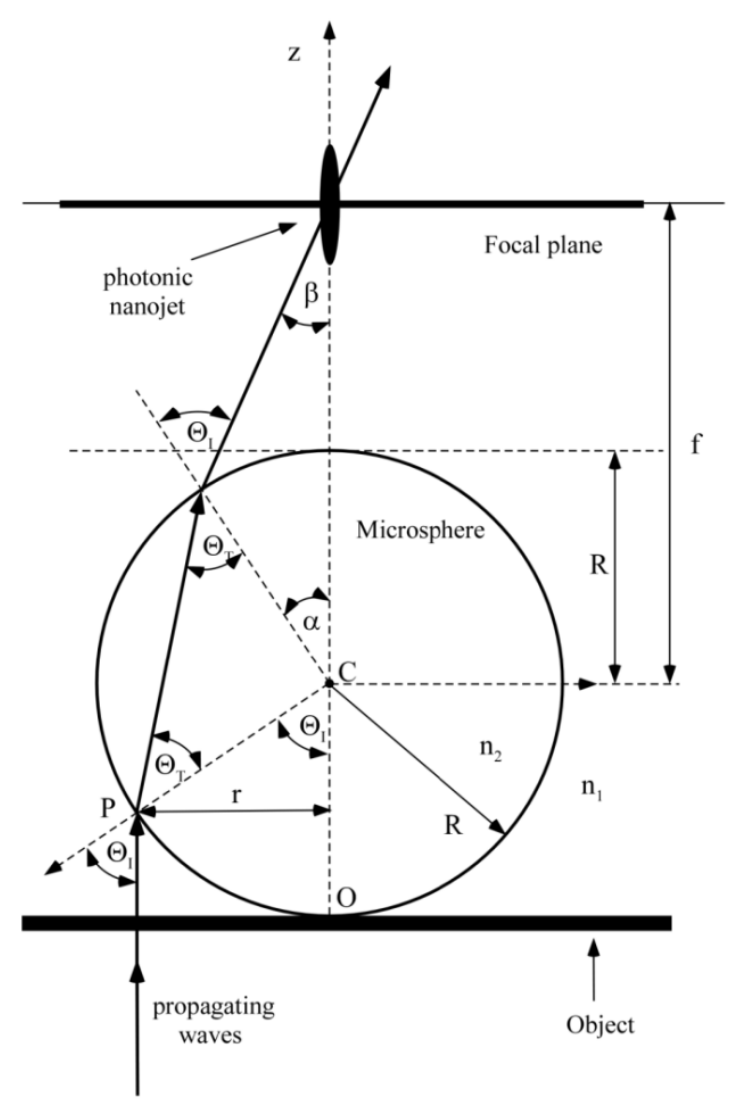

Figure 2 Bessel functions superposed on geometric optics trajectories. Using the description of geometric optics the plane EM beam is transmitted through a thin film of the object and incident on the microsphere (which has a radius $R$ ) with an incident angle $\theta_{I}$ and with a distance $r$ from the symmetric axis $z$. The EM ray is transmitted into the microsphere with transmittance angle $\theta_{T}$ and after traversing through the micros phe re crosses the symmetry axis with an angle $\beta$. For a relatively large distance $r$, the evanescent waves decay before entering the microsphere. The distance $f$ between the crossing point and the center of the microsphere $C$ is considered as a variable focal length which is a function of the incident angle $\theta_{I}$. The relation between $\theta_{T}$ and $\theta_{I}$ is fixed by the Snell law where the microsphere index of refraction is $n_{2}$ and outside it is $n_{1}$. The variable angle $\beta$ is calculated in the present article as a function of the incident angle. The approximation of physical optics for the nanojet is obtained by superposing Bessel beams on the trajectories of geometric optics.

Many possibilities have been suggested about the origin of super-resolution in microsphere experiments. It was claimed that the "super-resolution of a dielectric microsphere is governed by the waist of its photonic nanojet" [55]. Although this claim is correct, its interpretation should be related to the interference between the evanescent waves (converted to propagating waves) and nanojets. While the intensity of a nanojet might be very large relative to the evanescent waves (converted into propagating waves), the microsphere super-resolution is governed by photon changes $\Delta n$ of the evanescent waves. As the evanescent waves are concentrated near the symmetry axis such interference is effective only by the narrow waist of the nanojet, so that both waves are located at the same place. 
Super-resolution of a gold nanosphere located within a nanojet was reported [57]. Our assumption is that a nanojet alone without the evanescent waves (converted to propagating waves) cannot detect the fine structures of the object as they are not available to the nanojet and only a small fraction of structures can be detected directly by nanojets. The present approach is that the high resolution of microspheres is obtained by the interference of evanescent waves (converted to propagating waves) and nanojets have very important implications as detailed in the present work.

The accurate calculations of nanojets follow from the complicated solutions of Maxwell's equations by Mie theory [40-56] but such calculations do not give analytical results, as they require the summation of a large number of terms for a moderate sphere size. Further, optical resonances may also appear in micros pheres' photonic nanojets related to Mie terms in which the denominator is zero [53]. A more simple explanation of this effect and its approximate qualitative properties are described in Section 5.

The present paper is arranged as follows: In Section 2, we analyze the conversion of evanescent waves to propagating waves and show that this analysis is in agreement with experimental observations. We carried out the analysis for a simple example in the transmission mode but the same can also be done in the reflection mode and with more complicated examples. In Section 3, we show that microsphere super-resolution can be related to quantum cooperative effect between $n$ photons by which the effective wavelength is reduced to $\lambda / n$ and there is a distribution over different $n$ numbers. The relations between this effect and number-phase uncertainty relation is discussed. In Section 4, we show that plasmon interactions can be used for measuring fine structures of special systems. However, in a more general system, this interaction can change the optical image in a way that it becomes difficult to analyze, thus most microsphere super-resolution experiments are conducted without such interactions. The interference between evanescent waves and the nanojet near the focal is analyzed in Section 5, where this interference increases the intensity of light and leads to phase-contrast effects which are very important for fine structure measurements of the biological systems. There have been described many methods to increase the phase contrast in microsphere experiments by adding interference of the radiation transmitted or reflected by the microsphere with a reference system. These holographic systems have also been described in detail. In Section 6, we summarize our results and conclusions.

\section{Conversion of Evanescent Waves into Propagating Waves}

The microspheres act as a tip detector of SNOM where its geometry is not known but in our case, the microsphere has well-defined geometry which is used as follows. As described in Figure 1, a dielectric microsphere with refraction index $n_{2}$ and radius $R$ is located above a thin film of object at contact point $O$. The medium between the object and the microsphere has a refraction index $n_{1}$. Parallel monochromatic EM wave is transmitted through the thin object film in a direction perpendicular to its surface. We assume that the EM field in the medium with refractive index $n_{1}$ can be described by

$$
V(\vec{r}, t)=U(\vec{r}) \exp (i \omega t) \text {. (2) }
$$

where space dependent part $U(\vec{r})$ satisfies the Helmholtz equation

$$
\left(\Delta^{2}+k^{2}\right) U(\vec{r})=0 \quad ; \quad k=n_{1} k_{0} \quad ; \quad k_{0}=\frac{\omega}{c}
$$


where $k_{0}$ and $c$ are the wave vector and velocity of the monochromatic light in vacuum, respectively. The EM field at the upper planar surface of the object (for which: $z=0$ ) is given by

$$
U(x, y, z=0)=\int_{-\infty}^{\infty} \int_{-\infty}^{\infty} u\left(k_{x}, k_{y}\right) \exp \left[-i\left(k_{x} x+k_{y} y\right)\right] d k_{x} d k_{y} .
$$

where $k_{x}, k_{y}$ are the lateral spatial coordinates in the momentum space. The spatial structures of the object are included in the distribution of the spatial modes $u\left(k_{x}, k_{y}\right)$. The EM waves propagating from the surface of the object into the homogeneous medium in the space $z>0$ with a refractive index $n_{1}$ is given by

$$
U(x, y, z>0)=\int_{-\infty}^{\infty} \int_{-\infty}^{\infty} u\left(k_{x}, k_{y}\right) \exp \left[-i\left(k_{x} x+k_{y} y+k_{z} z\right)\right] d k_{x} d k_{y} .
$$

Eq. (5) obeys the boundary condition established in Eq. (5) and also satisfies the wave equation in the medium before the microsphere. We are interested here in evanescent waves for that the Helmholtz equation can be given as $k_{z}=\sqrt{k^{2}-k_{x}{ }^{2}-k_{y}^{2}}=-i \gamma\left(k_{x}, k_{y}\right)$. Notice that the decay constant $\gamma$ is given as a function of $k_{x}, k_{y}$. Eq. (5) applied for evanescent waves is transformed to

$$
\left.U_{\text {evan }}(x, y, z>0)=\int_{-\infty}^{\infty} \int_{-\infty}^{\infty} u\left(k_{x}, k_{y}\right) \exp \left[-i\left(k_{x} x+k_{y} y\right)-\gamma\left(k_{x}, k_{x}\right) z\right)\right] d k_{x} d k_{y} \cdot \text { (6) }
$$

For observing the fine structures of the object which are better than the Abbe resolution limit, we need to use such spatial modes, for which $k_{x}{ }^{2}+k_{y}{ }^{2}>k^{2}$ but under this condition, the waves are evanescent.

We simplify the analysis by taking into account the spherical symmetry of the microsphere. As described in Figure 1, a certain spatial mode of the evanescent EM waves is incident on the microsphere, at the surface point $P$ for which the sum of lateral spatial coordinates is $k_{I}$ in the $\mathrm{x}$ direction (i.e. $k_{x}=k_{I} ; k_{y}=0$ ) with $k_{I}^{2}>k^{2}$.

Then we have the spatial mode

$$
u(x, z)=\exp \left[-i k_{I} x-\gamma z\right] \quad ; \quad k_{I}^{2}-\gamma^{2}=k^{2}=n_{1}^{2} k_{0}^{2} \cdot(7)
$$

Here the subscript $I$ refers to the incident wave. The evanescent wave at the point $P$, which is above the object at a distance $h$, decays by factor $e^{-\gamma h}$ before arriving at this point so that only evanescent waves that are at a distance $r \ll R$ from the contact point $O$ are effective in producing the super-resolution effects.

Our basic idea is to use boundary condition for the light transmitted from the medium with a refractive index $n_{1}$ to another medium with the refractive index $n_{2}$. As is well known, the component of the monochromatic light wave vector, which is parallel to the boundary plane, is not changed by transmittance. We postulate that such boundary condition would also be valid for complex wave vector as analyzed in the previous paper [59]. The complex wave vector $\vec{k}_{\text {comp. }}$ incident on the microsphere at point $P$ can be described as $\vec{k}_{I, \text { comp. }}=k_{i} \hat{x}-i \gamma \hat{z}$.

The component of this complex wave vector, which is parallel to the microsphere surface at point $P$, is given by $\vec{k}_{I, \tan .}=k_{\tan } \hat{x}^{\prime}$ and it is not changed by transmission $\vec{k}_{I, \tan }=\vec{k}_{T, \text { tan. }}$ where $\vec{k}_{T, \tan }$. is the component of the complex wave vector that is tangential to the microsphere at point $P$ inside the microsphere. 
Let us analyze now, the conditions under which the evanescent waves are transformed into propagating waves. By rotating the spatial coordinates $\left(k_{I},-i \gamma\right)$ at point $P$ by the angle $\phi$ in the counter-clockwise direction, the spatial component $k_{I, \tan } \hat{x}^{\prime}$, which is tangential to the microsphere at this point, is given by

$$
k_{I, \tan }=k_{I} \cos (\phi)-i \gamma \sin (\phi) \text {. }
$$

where $k_{I, \tan }$ is a complex number. The boundary condition at point $\mathrm{P}$ leads to the relation

$$
k_{I, \tan }=k_{T, \tan } \cdot(9)
$$

where $k_{T, \text { tan }}$ is the tangential component of this spatial vector inside the microsphere at point $P$. The present spatial mode satisfies the Helmholtz equation in the microsphere with the relation

$$
k_{T, \tan }^{2}+k_{T, N}^{2}=n_{2}^{2} k_{0}^{2} \cdot(10)
$$

where $k_{T, N}$ is the component of the present spatial mode in the microsphere which is perpendicular to the microsphere surface at point $P$. We substitute Eqs. (8-9) into Eq. (10) and get:

$$
k_{T, N}^{2}=n_{2}^{2} k_{0}^{2}-k_{T, \tan }^{2}=n_{2}^{2} k_{0}^{2}-k_{I}^{2} \cos ^{2} \phi+\gamma^{2} \sin ^{2} \phi+2 i k_{I} \gamma \sin \phi \cos \phi .
$$

In Figure 1, we assumed that $k_{I}$ is in the $x$ direction but we have the same value of $k_{I}$ also in the $-x$ direction (following from the requirement that a standing wave is produced) so that the averaged value of $2 i k_{I} \gamma \sin \phi \cos \phi$ vanishes. Averaging this term to zero and substituting Eqs. (910) into Eq. (11) we get:

$$
k_{T, N}{ }^{2}=n_{2}{ }^{2} k_{0}^{2}-\left(n_{1}{ }^{2} k_{0}^{2}+\gamma^{2}\right) \cos ^{2} \phi+\gamma^{2} \sin ^{2} \phi=n_{2}{ }^{2} k_{0}^{2}-n_{1}{ }^{2} k_{0}^{2} \cos ^{2} \phi-\gamma^{2} \cos (2 \phi) .
$$

The evanescent wave is transformed into the propagating wave under the condition that $k_{T, N}{ }^{2}$ is positive but it will remain evanescent wave if its value is negative, thus in such case $k_{T, N}=-i \gamma_{\text {sphere }}$ where $\gamma_{\text {sphere }}$ represents the decay constant in the direction $z^{\prime}$ that is perpendicular to the microsphere surface. For small angles, we have approximate relations $\cos ^{2} \phi \simeq 1 ; \cos (2 \phi) \simeq 1$ and the condition for transforming evanescent waves to propagating waves can be given approximately by

$$
n_{2}{ }^{2} k_{0}{ }^{2}-n_{1}{ }^{2} k_{0}{ }^{2}-\gamma^{2}>0 \text {. (13) }
$$

The different properties of high resolution related to the above analysis have been discussed in previous works $[58,59]$ including the relation between the radius of the microsphere and the super-resolution. The use of microsphere as a tip detector for increasing the resolution by using scanning near-field optical microscopy (SNOM) of evanescent waves was related to complex Snell's law [58, 59].

In various experiments on microsphere high resolution, $n_{1}$ was taken approximately as 1 (air) and $n_{2}$ as around 1.5. However, it was emphasized in many works that the resolution can be improved further by using microspheres with high refraction index (nearly 2) [60-64]. These results can approximately be related to Eq. (13), or to the more general Eq. (12), by which more optimal condition of conversion of evanescent waves to propagating waves is given by $n_{2} \gg n_{1}$ Microspheres with liquid immersion were used, and the roles played by each component were studied [65-70]. One should notice that by increasing the value of $n_{1}$, the condition for converting evanescent waves to propagating waves becomes less optimal according to Eqs. (12-13). However, on the other hand, the Abbe resolution limit given by Eq. (1) improves (while the present 
improvement in the micro-sphere resolution is relative to the Abbe limit) [59]. Thus, the optimal condition for super-resolution is a tradeoff between these contrasting conditions [65-70].

Since the field of view in microsphere measurements is quite small, various methods have been used to get a large field of view in microsphere experiments. This includes moving the microsphere over the object or moving the object below the microsphere until the complete image is captured [71-77]. Super-resolution has been obtained by arrays of microspheres [78-82] and microfibers have also been used to increase the field of measurements [83, 84].

\section{Non-Classical Super-Resolution Effects in Microsphere Experiments and Number-Phase Uncertainty Relation}

Space dependent part of the monochromatic evanescent can be given in our treatment by exchanging Eq. (4) to the following Fourier series expansion [85]:

$$
\begin{aligned}
& U(x, y)=\left\{\sum_{m(1)=0}^{\infty} A_{m(1)} \exp \left(-i m(1) k_{0} x\right)+\sum_{m(2)=0}^{\infty} A_{m(2)} \exp \left(i m(2) k_{0} x\right)\right\} \\
& \times\left\{\sum_{n(1)=0}^{\infty} A_{n(1)} \exp \left(-i n(1) k_{0} y\right)+\sum_{n(2)=0}^{\infty} A_{n(2)} \exp \left(i n(2) k_{0} y\right)\right\} \\
& \times \exp \left(-\gamma_{m(i) n(j)} z\right)
\end{aligned}
$$

Eq. (14) includes Fourier series expansion of four-momentum modes. The terms in the first curly brackets represent linear combinations of momentum states propagating in $+x$ and $-x$ directions, while the terms in the second curly brackets represent momentum states propagating in the $+y$ and $-y$ directions. Here, $\mathrm{m}(1), \mathrm{m}(2), \mathrm{n}(1)$, and $\mathrm{n}(2)$ are the numbers of cooperative photon for the four modes, respectively. Further, $A_{m(i)}(i=1,2)$ and $A_{n(j)}(j=1,2)$ are the amplitudes of these modes. These amplitudes are obtained by using the boundary conditions at $z=0$. The decay of the evanescent waves (in the $z$-direction) is fixed by the relation:

$$
\left[m(i) k_{0}\right]^{2}+\left[n(j) k_{0}\right]^{2}-\left(\gamma_{m(i) n(j)}\right)^{2}=k_{0}^{2} \text { for } i, j=1,2 ; m(i)^{2}+n(j)^{2}>1 \text {. }
$$

For simplicity, we assumed the refraction index $n_{1}=1$ in Eqs. (14-15). Eq. (14) describes the discrete spectrum of EM waves by discrete spatial vectors. The quantization of this equation by using creation and annihilation operators in momentum space was developed in earlier work [14].

The non-classical behavior of super-resolution is related to the cooperation between $n$ photons increasing the wave vector to $n k_{0}$ and correspondingly decreasing the effective wavelength to $\lambda / n$ [86] and there is a distribution over different $n$ numbers. The role of coherence in microsphere assisted microscopy has been described earlier [87]. Here the nonclassical behavior of super-resolution is related to the quantum coherence between a large number of photons. We can describe this effect by taking the following example.

Cooperation between 5 photons which are propagating in the $x$ direction will decrease the effective wavelength to $\lambda / 5$. Such a wave is described as:

$$
U(x, y, z) \propto \exp \left(-i 5 k_{0} x\right) \exp \left(-4.9 k_{0} z\right) ;\left(5 k_{0}\right)^{2}-\left(4.9 k_{0}\right)^{2}=k_{0}{ }^{2} .(16
$$

A high resolution in the $x$ direction leads to the strong decay in the $z$ direction. By converting the evanescent wave to propagating wave, this decay disappears but the quantum coherence effect by which the effective wavelength is $\lambda / 5$. The quantum coherence effects are not changed 
by the transmission or reflection by the microsphere. In principle, there are many spatial modes in the evanescent waves with different photon numbers $n$ where the corresponding effective wavelength is reduced to $\lambda / n$.

The quantum effect that $n$ photons lead to an effective wavelength of $\lambda / n$ has an important implication related to the fact that the evanescent waves include the phases of the object. These phases can be related to number-phase uncertainty relation in a quantum mechanistic manner. Dirac was the first to quantify phases by an uncertainty relation $\Delta n \Delta \phi \geq 1 / 2$ following analogy of the well-known position-momentum uncertainty relation. Dirac's relation is problematic since it requires $\Delta \phi$ to be larger than $\pi$ for small $\Delta n$ so that complicated analyses on number phase uncertainty relations need to be carried out [88-89]. For the microsphere evanescent waves, the radiation phases are proportional to $1 / n$ (where $n>1$ and there is a distribution over different $n$ values) so that Dirac's relation can be used as a fair approximation.

The fluctuations in the cooperative number of photons lead to changes in radiation phases. This relation implies that one can use phase-contrast effects [32] to measure object phases especially to measure the phase objects. For a phase object in which the changes occur only in the thickness and index of refraction (e.g., certain semi-transparent biological tissue or cell), the conventional measuring systems cannot observe such changes as they measure light intensity (i.e., amplitudes) and not the phase changes. The phase-contrast measurements are based on the interference between the radiation transmitted (or reflected) by the object and another reference radiation system. Such interference converts phase changes into light intensity changes measured by the combined system. In microsphere high-resolution systems such phase-contrast occurs naturally in the system as the detectors measure the interference between the evanescent waves that include the phases of the object and the nanojets are composed mainly of on-evanescent waves. Although the microsphere system includes phase-contrast effects, for the better measurement of phase objects, it is recommended to use additional reference radiation system which will increase the phase contrast, as described in Section 5. Owing to the number-phase uncertainty relation, by increasing the cooperative radiation number $n$, the phases, which are smaller than those obtained in conventional phase contrast systems, can be measured (in equivalent explanation decreasing the effective wavelength to $\lambda / n$ enables us to measure smaller phases).

\section{Point Spread Function and Plasmon Interaction in Microsphere-Super-Resolution Measurements}

While we described in Section 2, the conversion of evanescent waves to propagating waves for a special system, there is a more general approach to super-resolution related to point spread function [90]. According to this approach [91], the spatial frequencies $F\left(k_{x}^{\prime}, k_{y}^{\prime}\right)$ measured by the detector of the microsphere system are given by the convolution of the object spatial frequencies $u\left(k_{x}, k_{y}\right)$ with the point spread function $B\left(k_{x}, k_{y}\right)$ of the optical system

$$
F\left(k^{\prime}{ }_{x}, k^{\prime}{ }_{y}\right)=u\left(k_{x}, k_{y}\right) * B\left(k_{x}, k_{y}\right) \text {. }
$$

where the symbol $*$ represents convolution and Eq. (17) can be written more explicitly as

$$
F\left(k_{x}^{\prime}, k_{y}^{\prime}\right)=\int_{-\infty}^{\infty} \int_{-\infty}^{\infty} u\left(k_{x}, k_{y}\right) B\left(k_{x}^{\prime}-k_{x}, k_{y}^{\prime}-k_{y}\right) d k_{x} d k_{y} .
$$


Different physical effects can lead to the point spread function that is obtained by the combination of these effects. As is well known, the use of metals with plasmon interaction can increase the transmission of EM waves by narrow slits [33-35] and increase the conversion of evanescent waves to propagating waves [36]. We discuss here the possibility to use plasmon interactions in microsphere experiments.

Most investigators agree that the original experiments conducted by Ebbesen et al. [33-35] are related to coupling the light with surface plasmon (SP). SP appears at the interface between a metal and dielectric material and has a combined EM wave and surface charge transfer. They have a transverse component magnetic field and two components for the electric field (TM modes), where the generator of surface charge requires an electric field normal to the surface. The SP fields are excited when the momentum of the surface plasmon is equal to the EM wave vector component that is parallel to the surface plasmon plus the reciprocal lattice vectors of the holes in the metal as explained in earlier works [33-35]. For an array of holes in a thin film, the matching condition is given by

$$
\vec{k}_{s p}=\vec{k}_{0 x} \pm n \vec{G}_{x} \pm m \vec{G}_{y} .
$$

Where $\vec{k}_{s p}$ is the SP wave vector and $k_{0 x}=(2 \pi / \lambda) \sin \theta$ is the component of the incident photons wave vector $\vec{k}_{0}$ in the plane of the grating. $\quad \vec{G}_{x}$ and $\vec{G}_{y}$ are the reciprocal lattice vectors where $\left|\vec{G}_{x}\right|=2 \pi / a_{0} ;\left|\vec{G}_{y}\right|=2 \pi / b_{0}, a_{0}$ and $b_{0}$ are the periods of the lattice and $m, n$ are integers. When the radiation is incident perpendicular to the plane of the grating $k_{0 x}=0$, the dependence of the momentum matching conditions on wavelength enters through $n \vec{G}_{x} \pm m \vec{G}_{y}$.

The enhancement of transmission in the experiments conducted by Ebbesen et al. [33-35] was based on previous work [36] on the convolution between the momentum transfer function of the slits and the momentum distribution function of $\vec{k}_{s p}$. One might note that the convolution between the momentum distribution function $\vec{k}_{s p}$ and the momentum distribution function $u\left(k_{x}, k_{y}\right)$ of Eqs. (4-5) can lead to the conversion of evanescent waves to propagating waves. In a study, microsphere assisted super-resolution optical imaging by plasmon interaction was reported [92]. Such interactions are, however, very complicated in general cases and can change the image in a way which is difficult to analyze. We notice that most experiments on microsphere superresolution were conducted with dielectric spheres without SP interactions.

\section{Interference of Evanescent Waves (Converted to Propagating Waves) with Nanojets Confined in Transversal and Axial Directions and Phase-Contrast Measurements}

Accurate calculations of nanojets follow from solutions of Maxwell equations by Mie theory. But such calculations do not give analytical results as they require summation of many complicated terms. The nanojets produced in microsphere super-resolution experiments can be described by the following simple approach explaining their main properties. We use geometric optics as the first-order approximation. Then the diffraction effects are related to Bessel beams superposed on the geometric optics trajectories.

As illustrated in Figure 2, EM wave is transmitted through the thin film of the object in a perpendicular direction and is incident on the microsphere at point $P$. For most EM beams 
entering the microsphere, the distance between the object and point $P$ is enough large so that the evanescent waves decay before entering the microsphere while the propagating wave remains $\left(k_{x}^{2}+k_{y}^{2}<n_{1}^{2} k_{0}^{2}\right)$. The incident angle $\theta_{I}$ and the transmitted angle $\theta_{T}$ are related by Snell's law $n_{1} \sin \theta_{I}=n_{2} \sin \theta_{T}$. As described in Figure 2, this wave is transmitted from the microsphere with opposite angles, i.e., with the incident and transmitted angles $\theta_{T}$ and $\theta_{I}$, respectively. The optical ray transmitted through the microsphere intersects the symmetric $z$ axis with angle $\beta$. The distance $r$ between the symmetry $z$ axis and the point $\mathrm{P}$ is given by $r=R \sin \left(\theta_{I}\right)$. We get the following relations:

$$
\theta_{I}-\alpha=\beta \quad ; \quad \theta_{I}+\alpha=2 \theta_{T} .(20)
$$

Then we get:

$$
\beta=2 \theta_{I}-2 \theta_{T} \text {. (21) }
$$

The distance $f$ between the center of the sphere and the crossing point on the symmetry axis is given by

$$
\frac{f}{\sin \theta_{I}}=\frac{R}{\sin \beta} \rightarrow f=\frac{R \sin \theta_{I}}{\sin \left[2 \theta_{I}-2 \theta_{T}\right]}=\frac{r}{\sin \left[2 \theta_{I}-2 \theta_{T}\right]}
$$

$f$ is a variable focal length which decreases as a function of the incident angle $\theta_{I}$ (or equivalently as a function of the distance $r$ of the point $P$ from the symmetry axis $\mathrm{z}$ ),

Assuming, for example $n_{2}=1.5 ; n_{1}=1$, we get $f \simeq 1.486 R$ for $\theta_{I}=10^{\circ}$, while for $\theta_{I}=45^{0}$ we get $f \simeq 1.27 R$. The value $f \simeq 1.486 R$ is approximately equal to the paraxial approximation [56] for the focal length given by $f=(n R / 2(n-1)=1.5 R$. Thus $f$ becomes smaller for larger values of $\theta_{i}$ for which the paraxial approximation is not valid. The variable $f$ is related to the optical depth already obtained from geometric optics but can be given via more rigorous calculations by Mie theory for the photonic jet. This optical depth is important for the interference of the evanescent waves (converted to propagating waves) and the nanojet in microsphere experiments.

One can use the geometric approach to get approximate results for the location of the image and its magnification by using the relation: $1 / a+1 / x=1 / f$ where $a$ is the distance of the object from the center of the microsphere $C$ and $x$ is the distance of the image from this point. Assuming that the object is below point C by the distance $R+\delta$, where $\delta$ is a small distance, we get $1 / x=1 / f-1 /(R+\delta) \rightarrow x=\frac{(R+\delta) f}{R+\delta-f}$. In case of most situations, $f>R+\delta$ then we get a negative value of $x$ with virtual image. The magnification is given by $M=\frac{x}{a}=x /(R+\delta)=\frac{f}{R+\delta-f}$. So that when $R+\delta-f$ is a very small number, the magnification becomes very large but is restricted by the field of view of the microscope. We find that the geometric optics can explain some important features of microsphere experiments.

Thus far, we considered the geometric optics description. The present idea is that we have to take into account the spherical symmetry by superposing Bessel beams on the trajectory of geometric optics. As the spherical Bessel beam depends on the angle $\beta$, we need to solve the physical optics wave function $\psi(\rho, z, t)$, in cylindrical coordinates [93-95]. For each value of $\beta$, the solution is given by

$$
\psi(\rho, z, t)=F(\rho, \beta) \exp \left[-i\left(k_{z} z-\omega t\right)\right] ; \rho=\sqrt{x^{2}+y^{2}} .
$$


The exponential function describes non-diffracted propagation in the $z$ direction for one Bessel beam, where $z$ is the distance from the focal plane of this wave, $\rho$ is the distance from the symmetric axis and $\beta$ is given by Eq. (21). Substituting Eq. (23) in the wave equation $\nabla^{2} \psi(\rho, z, t)=\left(1 / c^{2}\right)\left(\partial^{2} / \partial t^{2}\right) \psi(\rho, z, t)$ we get $[93,94]$.

$$
\frac{d^{2} F(\rho, \beta)}{d \rho^{2}}+\frac{1}{\rho} \frac{d F(\rho, \beta)}{d \rho}+\left(k^{2}-k_{z}^{2}\right) F(\rho, \beta)=0 \text {. }
$$

In this case, we used the azimuthal symmetry of the wave function including the relation $k^{2}=\omega^{2} / c^{2}$. The solution of Eq. (24) that has the bright core on the symmetric axis is proportional to the Bessel function of order 0 , so that [94].

$$
F(\rho, \beta) \propto(\rho k \sin \beta) ;(k \sin \beta)^{2}+k_{z}^{2}=k^{2} .
$$

The central bright core of the Bessel function has a radius [93]:

$$
\rho_{0} \simeq \frac{2.405}{k \sin \beta}=\frac{2.405}{k \sin \left[2 \theta_{i n}-2 \theta_{T}\right]} \text {. }
$$

Since we have rays with different crossing angle $\beta$, we need to use superposition of the solutions (23-25) with continuous values of $\beta$ and with different weight parameters [94]. The nanojet is obtained in such a qualitative description by the superposition of the different bright cores. The radius over incident angles corresponds approximately to the width of the nanojet [94]. However, more rigorous results are obtained by Mie theory calculations.

One should notice that the solutions given by Eqs. (23-26) correspond to the boundary condition by which the nanojet light is maximal on the symmetry axis. By engineering center covered microspheres [96-98], the boundary condition is changed and the contribution of high order Bessel functions becomes important. Such systems have been studied in detail and referred to as dark field micros copy [96-98].

As explained above in Section 3, the quantum interpretation of super-resolution by evanescent waves is related to cooperative effects between $n$ photons where the effective wavelength is reduced to $\lambda / n$ and there is a quantum distribution in the 2-D and/or 3-D object over various $n$ values. From the experimental point of view, it means that the evanescent waves include the phases of the objects (proportional to the optical distance, i.e., to the geometric path multiplied by the index of refraction which varies at different points of the object). In order to extract such information, one should use interference between the evanescent waves (converted to propagating waves) and light from a reference system. In the usual experiments on microspheres, we naturally have such interference between the evanescent waves and the nanojet, which is produced mainly from beams far from the contact point, for which the evanescent waves decay before entering the microsphere. The interference between the evanescent waves and the nanojets includes phase-contrast effects. Such effects are especially important for the visualization of biological matter where besides the changes in amplitude of the transmitted EM wave, changes also occur in the phase. Super-resolution measurements of different kinds of biological systems have been reported [99-101]. The combination of microsphere-assisted 2D imaging with low coherence phase-shifting interference microscopy was studied [102]. The phase-contrast imaging can be enhanced by using a beam splitter, which divides the EM wave to a part which is transmitted or reflected from the object and another part used as a reference beam. The interference between these two parts can be used for phase-contrast measurements. The 
quantitative phase imaging in biomedicine was presented in detail [103]. In cells and biological tissues, only changes in the thickness of objects and refraction index can be observed by phasecontrast measurements. Digital holography for quantitative phase-contrast imaging was developed, in which numerical hologram reconstruction was described [104, 105] and these methods were applied for phase-contrast imaging by microspheres [106, 107]. Microsphereassisted super-resolved Mirau digital holographic-microscopy has also been attempted [108, 109]. In the Mirau interferometry, the interferometric system consists of a single microscope objective, and the reference wave is obtained by reflection from a small mirror that is built inside the objective.

\section{Summary Discussion and Conclusion}

In the present work, we analyzed the relations between microspheres' super-resolution and the properties of evanescent waves transmitted or reflected by the microspheres. The fundamental condition for converting evanescent waves to propagation waves was derived from Eq. (12) with an approximation given by Eq. (13). It was shown that these equations are in agreement with experiments carried out using microspheres with high refraction index. The role played by microspheres with liquid immersed material was clarified by comparing the results with the Abbe resolution limit.

The evanescent waves enable us to observe the fine structures of the object which are better than the Abbe resolution limit since they have high spatial lateral modes for which, $k_{x}^{2}+k_{y}^{2} \gg k^{2}$. However, since the evanescent waves are transformed to propagating waves by the microspheres, such relation does not hold in the microspheres anymore so that the question arises how the high resolution is conserved? In Section 3, we explained that the evanescent waves introduce a quantum coherence between $n$ photons $(n>1)$ and there is a distribution over different $n$ numbers, which is preserved during the transmission or reflection by the microsphere. This distribution is described by Fourier series expansion of $U(x, y)$ given by Eq. (14). The decay of evanescent waves with momentum quantum numbers $m(i)$ and $n(j)(i=1$ or $2 ; j=1$ or 2$)$ is given by Eq. (15). The relation between this quantum effect and the number-phase uncertainty relation was discussed in Section 3.

As described in Section 4, a general relation between the lateral spatial modes of the object and those of the image is given by the convolution between the point spread function $B\left(k_{x}, k_{y}\right)$ and the object's lateral distribution $u\left(k_{x}, k_{y}\right)$ as given by Eqs. (17-18). There might be different mechanisms for producing the point spread function. The enhancement of radiation transmittance through arrays of narrow holes in thin metallic films was related to the experiments of Ebessen et al. [33-35], in order to couple light with surface plasmons (SP). As analyzed in previous work [36], such enhancement of radiation was related to the convolution between the evanescent momentum transfer function of the slits and the momentum distribution function of SP. It was shown in various experiments that SP can increase the super-resolution effects by the microspheres. As explained in Section 4, this effect can also be related to the convolution between spatial distribution of the evanescent waves and those of the SP. However, the relations between the object and its image obtained by this technique are quite complicated (including loss effects) and difficult to analyze. Such experiments were found to be useful for obtaining good images only 
in certain special cases. Most experiments on microsphere super-resolution were conducted without considering plasmon interactions.

The role played by the nanojet in microsphere super-resolution experiments is discussed in Section 5. The nanojet is produced near the focal plane where its width is smaller than the wavelength and this width remains unchanged for some wavelengths. While the evanescent waves propagate near the symmetry axis, the nanojet is produced mainly by EM waves, which are incident far from the symmetry axis and their evanescent waves decay before entering the microsphere. Although the intensity of a nanojet, which is proportional to the number of transmitted photons $n$ is much stronger than that of the evanescent waves. Super-resolution is obtained by changing $\Delta n$, i.e., the number of photons, and these changes are effected mainly by evanescent waves. The interference between the nanojet and the evanescent waves (converted to propagating waves) increases the light intensity of the image and leads to phase-contrast effects which are efficient for visualization of biological systems. Such interference is valid due to confining the nanojet to a small region so that it is located at the same place as that of the evanescent waves (converted to propagating waves).

As described in Figure 2, by using the approximations of geometric optics for the trajectories of the EM beams producing the nanojets, we get two fundamental outcomes: a) Crossing angle $\beta$ of the trajectory of geometric optics with the symmetry axis $z$ is given by Eq. (21). b) The variable focal distance $f$ between the center of the sphere $C$ and the crossing point on the symmetric axis is given by Eq. (22). The focal length $f$ decreases as a function of the incident angle $\theta_{I}$ and describes the optical depth in microsphere experiments, which is obtained in more rigorous calculations by the Mie theory. Using the trajectories of geometric optics, approximations were given to the location of the image and its magnification.

The solution by physical optics for the nanojet was given by superposing spherical Bessel beams on the trajectories of geometrics optics. The wave function solution for a certain incident angle $\theta_{I}$, with the corresponding crossing angle $\beta$, is given by Eqs. (23-26), where $F(\rho, \beta)$ is the azimuthal part of the wave function and in the $z$ direction, we have a non-diffracted propagating plane wave. In this condition, we used the spherical symmetry of the microsphere, where $\rho$ is the distance from the symmetry axis. For a general case for which we have a bright core on the symmetric axis, the solution for $F(\rho, \beta)$ is proportional to the Bessel function of order 0 , as given by Eq. (25). Since the incident angle $\theta_{I}$ with the corresponding crossing angle $\beta$ is changing as a function of the incident point on the microsphere, one may get a perfect outcome by the superposition of Bessel functions and changing it continuously as a function of the incident angle. An estimate of the width of the nanojet, which is in agreement with the experiment was given earlier [94] by using Eq. (26) with an average value for the parameter $\beta$. It is interesting to note that in darkfield microscopy where the center covers microspheres, the zeroth-order Bessel function should be exchanged to high order Bessel function solutions to satisfy different boundary conditions.

The interference between evanescent waves (converted to propagating waves) and the nanojet includes phase-contrast effects, which are important for the visualization of biological systems. The phase-contrast effects can be increased by using interference between the radiation transmitted or reflected by the microsphere and that of a reference EM beam. The visualization can then be performed by using holographic methods. 


\section{Author Contributions}

Y. Ben-Aryeh did all this work.

\section{Funding}

This research did not receive any specific grant from funding agencies in the public, commercial or not-for-profit sectors.

\section{Competing Interests}

The author has declared that no competing interests exist.

\section{References}

1. Lipson A, Lipson SG, Lipson H. Optical Physics. Cambridge: Cambridge Press; 2011.

2. Wang Z, Guo W, Li L, Luk'yanchuk B, Kahn A, Liu Z, et al. Optical virtual imaging at $50 \mathrm{~nm}$ lateral resolution with a white-light nanoscope. Nat Commun. 2011; 2: 218.

3. Yan Y, Li L, Feng C, Guo W, Lee S, Hong M. Microsphere-coupled scanning laser confocal nanoscope for sub-diffraction-limited imaging at $25 \mathrm{~nm}$ lateral resolution in the visible spectrum. ACS Nano. 2014; 8: 1809-1816.

4. Lai HSM, Wang F, Li Y, Liu L, Jia B, Liu L, et al. Super-resolution real imaging in microsphereassisted microscopy. PLOS ONE. 2016; 11; e0165194

5. Guo $H$, Han $Y$, Weng $X$, Zhao $Y$, Sui $G$, Wang $Y$, et al. Near-field focusing of the dielectric microsphere with wavelength scale radius. Opt Expr. 2013; 21: 2434-2443.

6. Lee S, Li L, Ben-Aryeh Y, Wang Z, Guo W. Overcoming the diffraction limit induced by microsphere optical nanoscopy. J Opt. 2013; 15: 125710.

7. Devilez A, Bonod N, Wenger J, Gerard D, Stout B, Rigeneault H, et al. Three-dimensional subwavelength confinement of light with dielectric microspheres. Opt Expr. 2009; 17: 20892094.

8. Zhou Y, Tang Y, Deng Q, Zhao L, Hu S. Contrast enhancement of microsphere assisted superresolution imaging in dark field microscopy. Appl Phys Expr. 2017; 10: 082501.

9. Maslov AV, Astratov VN. Optical nanoscopy with contact Mie-particles: Resolution analysis. Applied Phys Lett. 2017; 110: 261107.

10. Maslov AV, Astratov VN. Imaging of sub-wavelength structures radiating coherently near microspheres. Appl Phys Lett. 2016; 108: 051104.

11. Yang $Y$, Liu $H$, Yang $M$, Cui $B$, Zhang W. Dielectric sphere-coupled THZ super-resolution imaging. Appl Phys Lett. 2018; 113: 031105.

12. Chen $L$, Zhou Y, Li Y, Hong M. Microsphere enhanced optical imaging and patterning: From physics to applications. App Phys Rev. 2019; 6: 021304.

13. McLeod E, Arrold CB. Subwavelength direct-write nano-patte rning using optically trapped microspheres. Nat Nanotechnol. 2008; 3: 413-417

14. Ben-Aryeh Y. Increase of optical resolution by evanescent waves. Phys Lett A. 2004; 328: 306312. 
15. Hao X, Kuang C, Li X, Zhang H, Li Y. Microsphere based microscope with optical superresolution capability. Appl Phys Lett. 2011; 99: 203102.

16. Sundaram VM, Wen SB. Analysis of deep sub-micron resolution in microsphere based imaging. Appl Phys Lett. 2014: 105: 204102.

17. Upputuri PK, Pra manik M. Microsphere-aided optical microscopy and its application for superresolution imaging. Opt Commun. 2017; 404: 32-41.

18. Perrin S, Montgomery P, Lecler S. Super-resolution imaging within reach. Spie Opt Eng. 2019; 58: 050501.

19. Perrin S, LI H, Leong-Hoi A, Lecler S, Montgomery M. Illumination conditions in micro-sphereassisted microscopy. J Microsc. 2019: 274: 69-75.

20. Van Putten EG, Akbulut D, Bertolotti J, Vos WL, Lagendijk A, Mosk AP. Scattering length resolves sub-100nm structures with visible light. Phys Rev Lett. 2011; 106: 193905.

21. Hoang TX, Duan $Y$, Chen $X$, Barbastathis G. Focusing and imaging in microsphere-based microscopy. Opt Expr. 2015; 23: 12337-12353.

22. Huszka G, Yang H, Gijs MAM. Microsphere-based super-resolution scanning optical microscope. Opt Expr. 2017: 25: 15079-15092.

23. Ye R, Ye YH, Ma HF, Ma J, Wang B, Yao J, et al. Experimental far-field imaging properties of a diameter spherical lens. Opt Lett. 2013; 38: 1829-1831.

24. Lee JY, Hong BH, Kim WU, Min SK, Kim Y, Jouravlev MV, et al. Near-field focusing and magnification through self-assembled nanoscale spherical lenses. Nat Lett. 2009; 460: 498501.

25. Huszka G, Gijs MAM. Super-resolution optical imaging: A comparison. Micro Nano Engin. 2019; 2: 7-28.

26. Stanescu SL, Vilain S, Galieni V, Goh G, Karpinska K, Barbolina I, et al. Imaging with the superresolution microsphere amplifying lens (SMALL) nanoscope. IOP Conf Series: J Phys Conf Series. 2017; 902: 012014.

27. Migliozzi D, Giijs MAM, Huszka G. Microsphere-mediated optical contrast tuning for designing imaging systems with adjustable resolution gain. Sci Rep. 2018; 8: 15211.

28. Perrin S, Li H, Lecler S, Montogomery P. Unconventional magnification behavior in microsphere-assisted microscopy. Opt Laser Technol. 2019; 114: 40-43.

29. Li PY, Tsao Y, Liu YJ, Lou ZX, Lee WL, Chu SW, et al. Unusual imaging properties of superresolution microspheres. Opt Expr. 2016; 24: 016479-016486.

30. Duan $Y$, Barbastathis $G$, Zhang B. Classical imaging theory of a micro lens with superresolution. Opt Lett. 2013: 38: 2988-2990.

31. Ben-Aryeh Y. Super-resolution measurements related to uncertainty relations in optical and biological fluorescence systems. J Quant Spect. Radative Transfer 2013; 131: 43-51.

32. Murphy DB, Davidson MW. Fundamentals of light microscopy and electronic imaging. NY: Wiley. 2013.

33. Ebbesen TW, Lezec HJ, Ghaemi HF, Thio T, Wolff PA. Extraordinary optical transmission through sub-wavelength hole arrays. Nature. 1998; 391: 667-669.

34. Ghaemi HF, Thio T, Grupp DE, Ebbesen TW, Lezec HJ. Surface plasmons enhance optical transmission through subwavelength holes. Phys Rev B. 1998; 58: 6779-6782.

35. Barnes WL, Dereux A, Ebbesen TW. Surface plasmon subwavelength optics. Nature. 2003; 424 : 824-830. 
36. Ben-Aryeh Y. Transmission enhancement by conversion evanescent waves into propagating waves. Appl Phys B. 2008; 91: 157-165.

37. Allen KW, Farahi N, Li Y, Limberopoulos NL, Walker Jr DE, Urbas AM, et al. Overcoming the diffraction limit of imaging nanoplasmonics array by microspheres and microfibers. Opt Expr. 2015; 23: 24484-24496.

38. Farcau C. Metal-coated microsphere monolayers as surface plasmon resonance sensors operating in both transmission and reflection modes. Sci Rep. 2019; 9: 3683.

39. Tame MS, McEnery KR, Ozdemir SK, Lee J, Maier SA, Kim MS. Quantum plasmonics. Nat Phys. 2013: 9; 329-340.

40. Heifetz A, Kong SC, Sahakian AV, Taflove A, Backman V. Photonic Nano- jets. J Commput Theor Nanosci. 2009; 6; 1979-1992.

41. Zhu J, Goddard LL. Spatial control of photonic nanojets. Opt Expr. 2016; 24: 030444-030469.

42. Chen Z, Taflove A, Backman V. Photonic nanojet enhancement and backscattering of light by nanoparticles: A potential novel visible-light ultramicroscopy technique. Opt Expr. 2004: 12: 1214-1220.

43. Yang S, Taflove A, Backman V. Experimental verification at visible light wavelengths of the backscattering enhancement phenomenon of the photonic nanojet. Opt Expr. 2011; 19: 70847093.

44. Kim MS, Scharf T, Muhlig S, Rockstuhl C, Herzig HP. Engineering photonic nanojets. Opt Expr. 2011; 19: 10206-10222.

45. Luk'yanchuk BS, Paniagua-Dominguez R, Minin I, Minin O, Wang Z. Refractive index less than two: Photonic nanojets yesterday, today and tomorrow. Opt Expr. 2017; 7: 1820-1847.

46. Ferrand P, Wenger J, Devilez A, Pianta M, Stout B, Bonod N, et al. Opt Expr. 2008; 16: 69306940.

47. Lecler S, Takakura Y, Meyrueis P. Properties of a three-dimensional photonic jet. Opt Lett. 2005; 30: 2641-2643.

48. Geints YE, Zemlyanov AA, Minin OV, Minin IV. Systematic study and comparison of photonic nano-jets produced by dielectric microparticles in 2D- and 3D- spatial configurations. J Optics. 2018; 20: 065606.

49. Yang S, Wang F, Ye YH, Xia Y, Deng Y, Wang J, et al. Influence of the photonic nanojet of microspheres on microsphere imaging. Opt Expr. 2017; 25: 27551-27558.

50. Wu M, Chen R, Ling J, Chen Z, Chen X, Ji R, et al. Creation of a longitudinally polarized photonic nanojet via an engineering micros phere. Opt Lett. 2017; 42: 1444-1447.

51. Duocastella M, Tantussi F, Haddadpour A, Zaccaria RP, Jacassi A, Veronis G, et al. Combination of scanning probe technology with photonic nanojets. Sci Rep. 2017; 7: 3474.

52. Lecler S, Perrin S, Leong-Hoi A, Montgomery P. Photonic jet lens. Sci Rep. 2019; 9: 4725.

53. Lee S, Li L, Wang Z. Optical resonances in microsphere photonic nanojets. J Optics. 2014; 16: 15704

54. Geintz YE, Zemlyanov AA, Panina EK. Photonic jets from resonantly excited Transparent dielectric microspheres. J Opt Soc Am B. 2012: 29: 758-762.

55. Yang $H$, Trouillon R, Huszka G, Gijs MAM. Super-resolution imaging of a dielectric microsphere is governed by the waist of its photonic nanojet. ACS Public Nano Lett. 2016; 16: 4862-4870.

56. Kofler J, Arnold N. Axially symmetric focusing as a cupsoid diffraction catastrophe: Scalar and vector cases, and comparison with the theory of Mie. Phys Rev B. 2006; 73: 235401. 
57. Heifetz A, Simpson JJ, Kong SC, Taflove A, Backman V. Sub-diffraction optical resolution of a gold nano-sphere located within the nanojet of a Mie-resonant dielectric microsphere. Opt Expr. 2007; 15: 17334-17342.

58. Ben-Aryeh Y. Super resolutions observed from evanescent waves transmitted through nanocorrugated metallic films. Appl Phys B. 2012; 109: 165-170.

59. Ben-Aryeh Y. Increase of resolution by use of microspheres related to complex Snell's law. J Opt Soc Am A. 2016; 33: 2284-2288.

60. Lee S, Li L. Rapid super-resolution imaging of sub-surface nanostructures beyond diffraction limit, by high refractive index microsphere optical nanoscopy. Optics Commun. 2015; 334: 253-257.

61. Darafsheh A, Walsh GF, Negro LD, Astratov VN. Optical super esolution by high- index liquidimmerse microspheres. Appl Phys Lett. 2012; 101: 141128

62. Guo M, Ye YH, Hou J, Du B. Size-dependent optical property of high-index immersed microsphere lens. Appl Phys B. 2016; 122: 65.

63. Guo M, Ye YH, Hou J, Du B. Experimental far-field imaging properties of high Refractive index microsphere lens. Photon Res. 2015; 3: 339-342.

64. Zhu H, Yan B, Zhou S, Wang Z, Wu L. Synthesis and super-resolution imaging performance of a refractive-index-controlling microsphere superlens. J Mat Chem C. 2015; 3: 10907-10915.

65. Darafsheh A, Limberopoulos NI, Derov JS, Walker Jr. DE, Astratov NV. Advantages of microsphere-Assisted super-resolution imaging technique, over solid immersion lens and confocal microscopies. Appl Phys Lett. 2014: 104: 061117.

66. Pang H, Cao A, Du C, Qiu Q, Deng O, Yin S. Spectrum analysis of liquid immersion to transparent microsphere based optical nanoscopy. Optik. 2015: 126: 3079-3083.

67. Ling J, Wang X, Li D, Liu X. Modeling and verification of white light immersion microsphere optical nanoscope. Opt Quant Electronics. 2017; 49: 377.

68. Lee S, Li L, Wang Z, Guo W, Yan Y, Wang T. Immersed transparent microsphere magnifying sub-diffraction-limited objects. Applied Optics. 2013; 52: 7265-7270.

69. Wang F, Yang S, Ma H, Shen P, Wei N, Wang M, et al. Microsphere-assisted super-reslution imaging with enlarged numerical aperture by semi-imersion. Appl Phys Lett. 2018: 112; 23101.

70. Zhou Y, Tang Y, He Y, Liu X, Hu S. Effects of immersion depth on super-resolution properties of index-different microsphere-assisted nanoimaging. Applied Phys. 2018; 11; 032501.

71. Allen KW, Farahi N, Li Y, Limberpoulos NI, Walker Jr DE, Urbas AM, et al. Super-resolution microscopy by movable thin-films with embedded microspheres: Resolution analysis. Annal Phys. 2015: 527; 513-522.

72. Darafsheh A. Comment on "Super-resolution microscopy by movable thin-films with embedded microspheres: Resolution analysis". Ann Phys. 2016; 528: 898-900.

73. Allen KW, Li Y, Astratov VN. Reply to "Comment on 'Super- resolution Microscopy by movable thin- films embedded in microspheres: Resolution analysis". Annalen der Physik. 2016: 528: 11-12.

74. Krvitsky LA, Wang JJ, Wang Z, Luk'yanchuk B. Locomotion of microspheres for super resolution imaging. Sci Rep. 2013: 3: 3501.

75. Mora CAG, Hartelt M, Bayer D, Aeschlimann M, Ilin EA, Oesterschulze E. Microsphere-based cantilevers for polarization- resolved and femtosecond SNOM. Appl Phys B. 2016; 122: 86. 
76. Du B, Ye YH, Hou J, Guo M, Wang T. Sub-wavelength image stitching with removable microsphere-embedded thin film. Appl Phys A. 2016; 122: 15.

77. Wang S, Zhang D, Zhang H, Han X, Xu R. Super-resolution optical microscopy based on scannable cantilever-combined microsphere. Microsc Res Techniq. 2015; 78: 1128-1132.

78. Darafsheh A, Guardiola C, Palovcak A, Finlay JC, Carabe A. Optical super- resolution imaging by high-index microspheres embedded in elastometers. Opt Lett. 2015; 40: 5-8.

79. Huszka G, Gijs MAM. Turning a normal microscope into a super-resolution instrument using a scanning microlens array. Sci Rep. 2018; 8: 601.

80. Allen KW, Darafsheh A, Abolmaali F, Mojaverian N, LImberopoulos NI, Lupo A, et al. Micros phere-chain waveguides: Focusing and transport properties. Appl Phys Lett. 2014; 105: 021112.

81. Allen KW, Abolmaali F, Duran JM, Ariyawansa G, Limberopoulos NI, Urbas AM, et al. Increasing sensitivity and angle-of-view of mid-wave infrared detectors by integration with dielectric microspheres. Appl Phys Lett. 2016; 108: 241108.

82. Wang T, Kuang C, Hao X, Liu X. Subwavelength focusing by a microsphere array. J Opt. 2011; 13: 035702.

83. Nolvi A, Laidmae I, Maconi G, Heinamaki J, Haeggstrom E, Kassamakov I. Wide field of view 3D label-free super-resolution imaging. Proc Spie. 2018: 10539: 1053912.

84. Astratv VN, Maslov AV, Allen KW, Farahi N, Li Y, Brettin A, et al. Fundamental limits of superresolution microscopy by dielectric microspheres and microfibers. Proc Spie. 2016; 9721: 97210K.

85. Ben-Aryeh Y. Evanescent waves and super-resolution effects. Int J Quant Inform. 2005; 3: 111115

86. Ben-Aryeh Y. Nonclassical high resolution optical effects produced by evanescent waves. J Opt B: Quant Semiclass Opt. 2003; 5: S553-S556.

87. Perrin S, Lecler S, Leong-Hoi A, Montgomery C. Role of coherence in Microsphere-assisted nanoscopy. Proc Spie. 2017: 10330: 103300V.

88. Brif C, Ben-Aryeh Y. Phase-state representation in quantum optics. Phys Rev A. 1944; 50: 3505-3516.

89. Opatrny T. Number phase uncertainty relations. J Physics A: Math General. 1995: 28: 6961.

90. Maslov AV, Astratov VN. Resolution and reciprocity in Micro-spherical nanoscopy: Point spread function versus photonic nano-jets. Phys Rev Appl. 2019: 11: 064004.

91. Ben-Aryeh Y. Tunnelling of evanescent waves into propagating waves. Appl Physics B. 2006: 84: 121-124.

92. Hou B, Xie M, He R, Ji M, Trummer S, Fink RH, et al. Microsphere assisted super resolution optical imaging of plasmonic interaction between gold nanoparticles. Sci Rep. 2017; 7: 13789

93. McGloin D, Dholakia K. Bessel beams: Diffraction in a new light. Contempor Phys. 2005; 46: 15-28.

94. Ben-Aryeh Y. Nan-jet related to Bessel beams and to super-resolutions in microsphere optical experiments. EPJ Techniq Instrument. 2017; 4: 3.

95. Gori F, Guattari G, Padovani C. Bessel-gauss beams. Optics Commun. 1987; 64: 491-495.

96. Yan B, Yue L, Wang Z. Engineering near-field focusing of a microsphere lens with pupil masks. Optics Commun. 2016; 370: 140-144. 
97. Perrin S, Li H, Badu K, Comparon T, Quaranta G, Messaddeq N, et al. Transmission microsphere-assisted dark-field microscopy. Phys Stat Sol. 2019: 13, 180045.

98. Wu M, Chen R, Soh J, Shen Y, Jiao L, Wu J, et al. Super-focusing of center-covered engineered microsphere. Sci Rep. 2016; 6: 31637.

99. Li L, Guo W, Yan Y, Lee S, Wang T. Label-free super-resolution imaging of adenoviruses by submerged microsphere optical nanoscopy. Light: Sci Appl. 2013; 2: e104.

100. Yang H, Moullan N, Auwerx J, Gijs MA. Super-resolution biological microscopy using virtual imaging by a microsphere nanoscope. Small. 2014; 10: 1712-1718.

101. Astratov VN, Maslov AV, Brettin A, Bianchette KF, Nesmelov YE, Limberopoulos NI, et al. Contact microspherical nanoscopy: From fundamentas to biomedical I applications. Spie Bios. 2017; 10077: 100770S.

102. Perrin S, Leong-Hoi A, Lecler S, Pfeiffer P, Kassamakov I, Nolvi A, et al. Microsphere-assisted phase-shifting profilometry. Appl Opt. 2017: 56: 7249-7255.

103.Park YK, Depeursinge C, Popescu G. Quantitative phase imaging biomedicine. Nat Photon. 2018; 12: 578-589.

104.Cuche E, Bevilacqua F, Depeursinge C. Digital holography for quantitative phase-contrast imaging. Opt Lett. 1999; 24: 291-293.

105. Mann CJ, Yu L, Lo CM, Kim MK. High-resolution quantitative phase-constrast microscopy by digital holography. Opt Expr 2005; 13; 8693-8698.

106.Wang Y, Guo S, Wang D, Lin Q, Rong L, Zhao J. Resolution enhancement phase-contrast imaging by micros phere digital holography. Optics Commun. 2016; 366: 81-87.

107.Abbasian V, Rasouli S, Moradi AR. Microsphere-assisted self-referencing digital holographic microscopy in transmission mode. J Opt. 2019; 21: 045301.

108. Aakhte M, Abbasian V, Akhlaghi EA, Moradi AR, Anand A, Javidi B. Microsphere-assisted super-resolved Mirau digital holographic microscopy for cell identification. Appl Opt. 2017; 56: D8-D13.

109. Kassamakov I, Lecler W, Nolvi A, Leong-Hoi A, Montgomery P, Haeggstrom E. 3D superresolution optical profiling using microsphere enhanced Mirau interferometry. Sci Rep. 2017; 7: 3683.

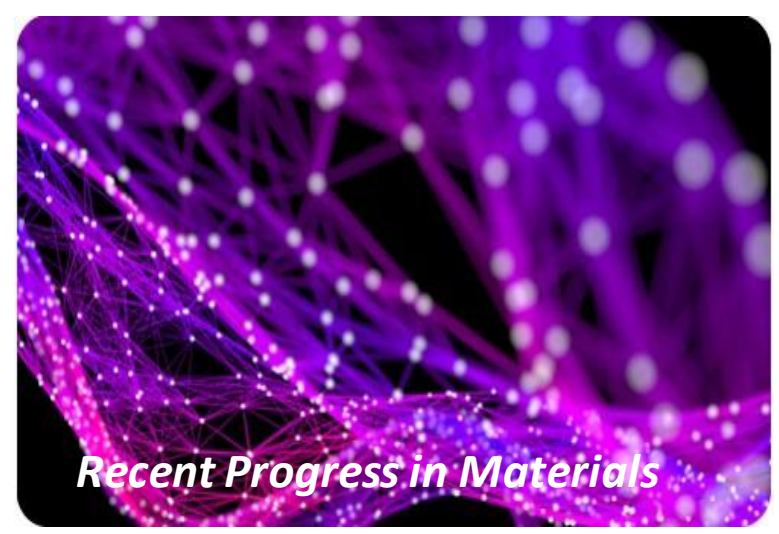

Enjoy Recent Progress in Materials by:

1. Submitting a manuscript

2. Joining in volunteer reviewer bank

3. Joining Editorial Board

4. Guest editing a special issue

For more details, please visit:

http://www.lidsen.com/journals/rpm 